Témoigner Témoigner. Entre histoire et mémoire

Getuigen Revue pluridisciplinaire de la Fondation Auschwitz

$126 \mid 2018$

Questions sur l'avenir du travail de mémoire

\title{
Questions sur l'avenir du travail de mémoire
}

Présentation

Vragen over de toekomst van de herinnering. Inleiding

Anneleen Spiessens et Frank Schroeder

(2) OpenEdition

Journals

Édition électronique

URL : https://journals.openedition.org/temoigner/7127

DOI : $10.4000 /$ temoigner.7127

ISSN : 2506-6390

Traduction(s) :

Vragen over de toekomst van de herinnering - URL : https://journals.openedition.org/temoigner/7133 [nl]

Éditeur :

Éditions du Centre d'études et de documentation Mémoire d'Auschwitz, Éditions Kimé

Édition imprimée

Date de publication : 2 avril 2018

Pagination : 47-53

ISBN : 978-2-930953-06-9

ISSN : 2031-4183

Référence électronique

Anneleen Spiessens et Frank Schroeder, «Questions sur l'avenir du travail de mémoire », Témoigner. Entre histoire et mémoire [En ligne], 126 | 2018, mis en ligne le 24 janvier 2022, consulté le 04 février 2022. URL : http://journals.openedition.org/temoigner/7127 ; DOI : https://doi.org/10.4000/temoigner. 7127

Tous droits réservés 
(Le colloque)

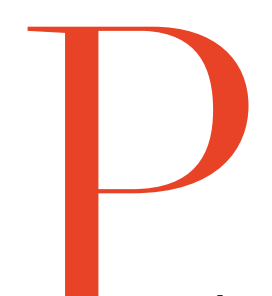

__ our le Musée national de la Résistance, petite institution située à Esch-sur-Alzette, au sud du GrandDuché de Luxembourg, le contexte du paysage memoriel est spécifique : au cours des dernières années, celui-ci été complètement bouleversé, suite l'émergence d'associations et d'initiatives privees comme MemoShoah asbl mais surtout grâce à la volonté politique de lui donner une nouvelle orientation et la tentation de calmer des tension entre différents groupes de victimes d nazisme. Trois centres de documentation ont fermé et de nouvelles structures ont été creees, comme le Comité de la Mémoire de la Deuxième Guerre mondiale, regroupant désormais les résistants, les enrôlés de force dans la Wehrmacht et la communauté juive. La recherche historique a été confiée au nouvel Institut d'histoire du temps présent/Luxembourg Center for Contemporary and Digital History, à l'Université du Luxembourg, qui a repris une partie du personnel des centres de recherch dissouts. Pour superviser la mémoire et la commemoration, un Service de mémoire a été cree au ministère d'État. Au milieu de tous ces remaniements, le musée d'Esch, qui a fêté son $60^{\mathrm{e}}$ anniversaire en 2016
En 2016, au Grand-Duché, des institutions et un comité étaient don en train de s'inventer, de définir leur tâches et approches futures. Il fallai par ailleurs tenir compte d'un musée vieillissant pour lequel la décision d’un renouvellement et d'un agrandissement venait juste d'être prise. C'étai pour celui-ci une période dintense questionnements et d'élaboration de nouveaux concepts.

C'est dans ce cadre que nous avon écidé d'organiser un col national afin de lancer une discussion publique sur l'avenir du travail de mémoire. Nous voulions que le débat dépasse le Luxembourg - dans l'esprit des reseaux et des partenariats qui $s$ étaient constitués au cours des dernières annees dans la grande région (Luxembourg, Wallonie, Lorraine, Sarre et Rhénanie-Palatinat), dans l'esprit du projet Interreg "Land of Memory ». Nous avions lambition que ce colloque puisse être «d'utilité publique », et qu'il devienne un evénement participatif, permettant à des specialistes et à des citoyens engages de discuter ensemble et de dessiner des pistes pour l'aveni de la mémoire - ou plutôt du travail de mémoire - axées sur l'éducation à la citoyenneté.

La palette impressionnante de spécialistes - d'un côté des scientifiques et chercheurs, de l'autre des praticiens lagrande diversité des sujets présentés, la qualité des discussions ainsi que le nombre surprenant de visiteurs - entre 120 et 150 par panel - ont contribué à la haute qualite du colloque. Frank Schroeder Directeur du Musée nationa de la Résistance d'Esch-sur-Alzette
La place de la Shoah dans notre culture mémorielle

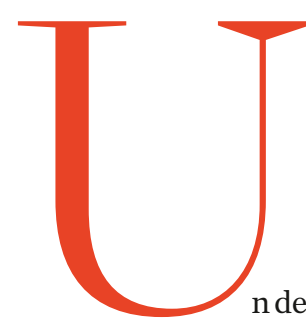
Jufin européens, 800000 Tutsis et Hutus modérés sont tués a Rwanda en l'espace d’à peine trois mois. Confrontée à l'explosion d'une violence quelle pense à plusieurs égards similair à celle qu'elle a elle-même subie, Simone Veil se remémore sa propre experience. Lors d'un entretien croise avec Esthe Mujawayo, rescapée rwandaise, ell avoue être atterrée par la situation a Rwanda

Pour moi, le Rwanda, c'est une page particulièrement douloureuse de toute ces années, depuis le retour du camp. Nous avions vraiment esperé qưune telle barbarie ne se reproduirait pas...'

peu près à la même époque, Innocent Rwililiza, lui aussi rescapé d génocide rwandais, confie au reporte français Jean Hatzfeld :

J'ai lu qưaprès chaque génocide les historiens expliquent que ce sera le dernier.Parce que plus personne ne pourr accepter une pareille infamie. Voilà une blague étonnante ${ }^{2}$.

On le sait désormais : génocide se décline au pluriel. La Shoah n'était n

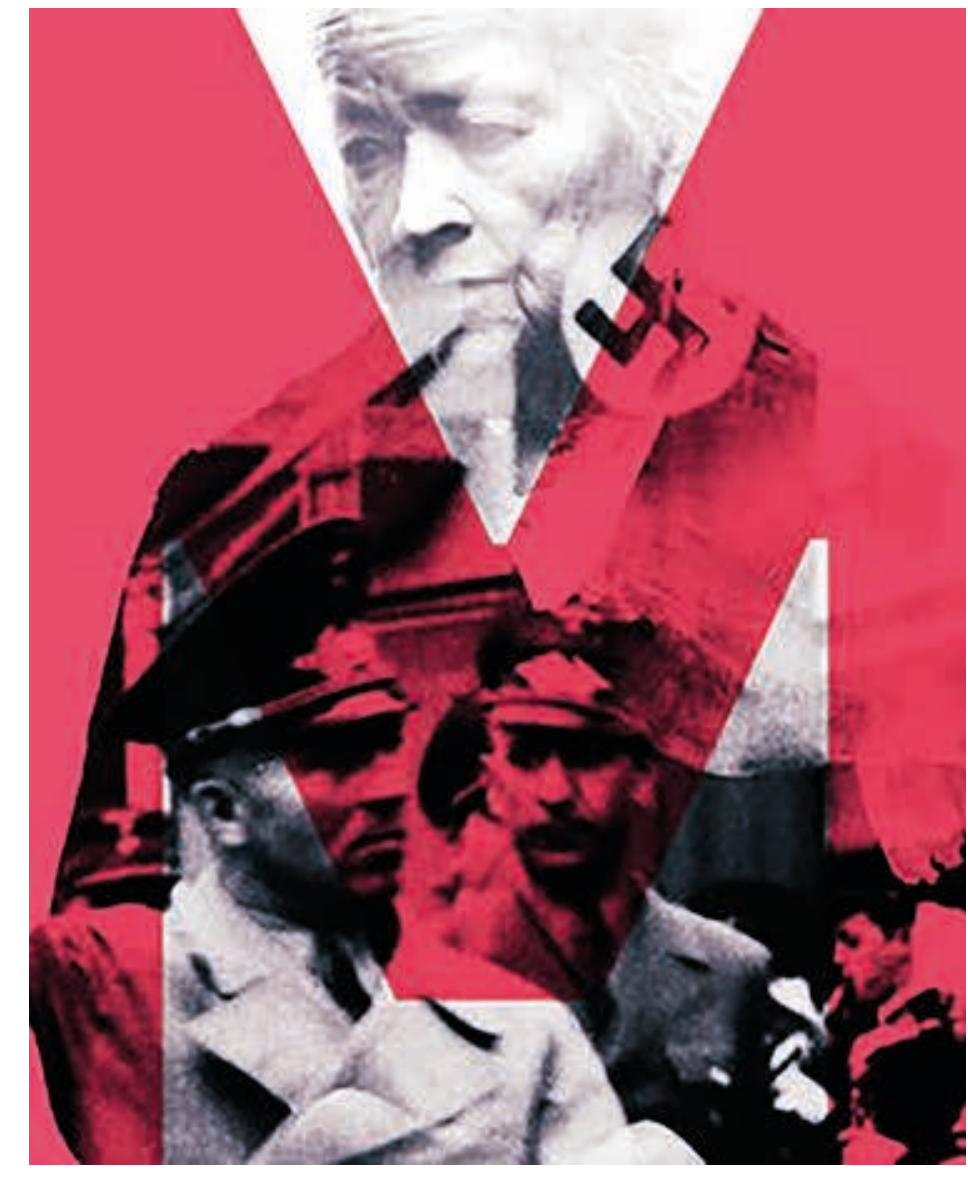

e premier ni le dernier du XXe siècle. Néanmoins, elle continueà fonctionner comme «paradigme» de la folie exterminatrice, de par sa portée symbolique et interprétative Cortée symbolique et ine Couro : Conne lanime Cathecreuset de méditation sitz restera un linhum un ditation sur lesconfins de des possibilités délirantese-repoussoir tisme allementet estesdelantisenitisme allemand et européen, et la biopolitique de l'État-nation moderne. »" auteure preconise notammentle développement d'une mémoire critique qui e fois stérile du « devoir de mémoire ».
(1) Esther Mujiawayo et Souâd
Belhaddad, SurV Vivantes, Paris,
Eddidions (2) Jean Hatzfeld, Dans le nu de Paris, Seuil, 2000, p. 105.

(3) « Du malentendu w, in des camps, penser les génocides,
Paris, Albin Michel, 1999, p. 55. 
Heidemarie Uhl le démontre parfaitement dans le premier article de ce dossier : la culture mémorielle qui s'est développée au milieu des années 198 en Europe s’avère propice à l'élaboration d'une telle mémoire critique. Les «mythes de l'après-guerre », ces récit nationaux focalisés sur la figure $d$ peuple oppressé ou celle du héros résistant, ont commence alors à s'éroder. La nouvelle génération (la generation of memory suivant la formule de Jay Winter) a bien compris que la mémoire, elle veut etre le fondement d'une poltique des droits de l'Homme et de la solidarité, seratransnationale. Etc'estle souvenir de la Shoah qui constituera le point d'ancrage de cette nouvelle culture mémorielle globale. La «Déclaration de Stockholm », adoptée lors du Stockholm International Forum on the Holocaust en 2000, souligne l'importance de lenseignement de lHolocauste afin de lutter aujourd hui contre le génocide, le nettoyage ethnique, le racisme, l'antisé mitisme et la xénophobie. Les 31 État membres de l'International Holocaus Remembrance Alliance (IHRA) chargent d'appliquer ces principes.

La «dénationalisation » de la mémoire de la Shoah s'oppose alors larticulation «nationale » de la question des Justes, theme repris par Françoi Wisard dans le contexte suisse. Effectivement, la notion de « Juste suisse parait peu pertinente pour désigner des personnes de double nationalité, des sauveteurs dont la nationalité changé au cours du temps, qui sont originaires de pays dont les frontiere ont été modifiees, ou qui sont honorées pour des actions réalisees dans un autre pays. Toutefois, le thème des Justes, et plus genereralement celui du sauvetage des Juils, occupe aujourd hui une place importante dans le travail de mémoir de la Shoah en Suisse, en témoigne l'essor de matériels pédagogiques élaborés ces dernières annees. François Wisard confirme que lacte de courage altruiste dont le Juste est le symbole « invite le élèves à réfléchir sur les choix possibles, sur une marge de manouvre - probablement plus étendue qu'on ne pourrai l'imaginer - dans des situations tragiques.» En même temps, il nous meten garde contre les pièges liès àl'utilisation de la figure du Juste. En se focalisant sur les «grandes figures exemplaires », mais pas forcènent «reprèsentatives», telle Carl Lutz ou Paul Grüniger en Suisse, on risque de présenter aux élèves un idéal trop « heroíque » qui reste inatteignable, ou encore de faire l'impasse sur la dimension collective de certaine actions.

La mémoire de la Shoah occupe aujourd'hui une place centrale dan les questions memorielles, mais la reconnaissance de cette memoire fut loin d'être une évidence - et ne l'est toujours pas - en Israēl. Gideon Grei nous rappelle que la Shoah a certes « toujours accompagné et marqué la société israélienne depuis la période précédant les jours de la création de lÉtat ", les survivants immigrés on pourtant longtemps été marginalisés. Le comportement des Juifs européen. victimes du nazisme, " conduits comme des moutons àlabattoir » selon certains, ne correspondait pas aux valeurs fondamentales de la societé israélienne. La guerre israélo-arabe de 1948 a davantage relégué la question du trauma des survivants à larrière-plan. Ce n'es qu'en 1961, avec le procès de Adol Eichmann, que les Israeliens prennent «vraiment » connaissance de l'histoire de la persécution et de l'extermination des Juifs d'Europe. La réflexion de Gideon Greif à ce propos fait écho aux

observations d'Annette Wieviorka qui, dans son ouvrage L'Ère du témoin ${ }^{4}$, soutient que le procès Eichmann marque un véritable tournant dans l'émergence de la mémoire du génocide en raison de l'instrumentalisation du témoignage. En effet, en parallèle à l'intensification du travail historiographique (le prestige de ce type de recherche dans les universités) et institutionnel ('importance de Yad Vashem), un corpus de témoignage se développe en Israël. Il permet aux survivants de finaloment participer au débat public et aux nouvelles générations de construire un rapport personnel avec ce passé douloureux

\section{Témoiginage et \\ transmission}

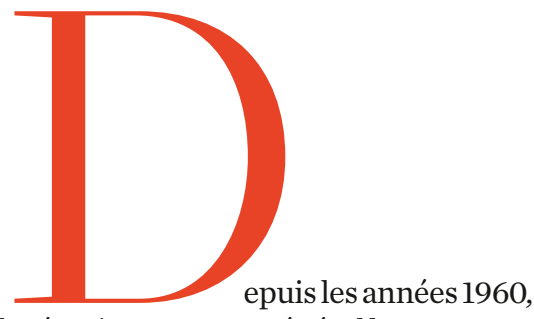

rallarencontre avec les témoins, constitue donc un élément essentiel du travail de mémoire de la Shoah. Il devient le dispositif préféré de la transmission, du « passage ». Grâce à son récit partiel, hésitant et souvent lacunaire, le témoin survivant, voilà bien le principe de base, « donne un visage » a levénement traumatique et, en faisant appel à l'empathie, provoque une prise deconscience aupres du grand public. Il est inquietant, dès lors, de constater avec Gideon Greif que les derniers temoins directs disparaissent. Leur absence a été anticipée par les campagnes d'enregistrement vidéo des annees 1990 et 2000, initiées avec le projet Fortunoff de Geoffrey Hartman a Yale, puis reprises par la Fondation Spielberg. Pourtant, il semble qu'il y ait autre chose en jeu que la seule disparition des témoins.

Revenons à Simone Veil et à sa réaction par rapport aux événements du Rwanda. Face à un nouveau génocide malgre cinquante ans de commémoelle exprime ses doutes sur l'utilité du témoignage qui, plutôt que marquer ne rupture, semble entrer dans une 
logique de continuité. On retrouve le même raisonnement chez Philip Gourevitch, journaliste américain et auteur d'un livre sur le Rwanda, qui se souvient de sa visite a l'US Holocaust dant qu'il attend son tour dans la file qu caractérise toute attraction touristique, son regard tombe sur une photo à la Un du journal - des cadavres boursouflés flottant dans une rivière

Un groupe d'employés du musée arriv pour prendre son travail. Sur leur blazer marron plusieurs portaient les insignes, vendus un dollar pièce à boutique du musée, frappés des slogan «Souvenez-vous » et « Jamais plus ». Le musée navait qu'un an; lors de so inauguration, le président Clinto lavait présenté comme " un investissement dans lavenir sûr contre tout folie future. » Apparemment, il voula simplement dire que les victimes d'exterminations a venir pouvaient desormais mourir paisiblement, en sachan qu'un sanctuaire existait a Washington où leurs souffrances pourraient être commémorées, mais à l'époque ces paroles semblaient riches d'une promesse plus hardie

La culture mémorielle est censé constituer un moyen préventif pour lutter contre les systèmes autoritaires et racisme, mais leeclatement de violence ethnique au Rwanda, puis en ex-Yougos lavie, nous oblige à repenser les fondements du travail de mémoire. En 2014 Philippe Mesnard soulevait déjà le problème dans l'édito de notre revue : capés (1985), a constaté la faillite de la ansmission testimoniale qui perdait, ar sa forme stéréotypée, de sa pertience dans le monde contemporain. C’est là « le véritable problème de notre âge $»^{6}$, une question qui traverse comme un fil rouge les deux derniers articles de ce dossier. En effet, qui transmet quoi et comment?

Yves Lapid, responsable du déparement pédagogique du Mémorial du camp de Mauthausen de 2007 à 2013, sintéresse à la dynamique des visite des lieux de mémoire. Plus particulièrement, il met en cause l'efficience du parcours traditionnel où le guide tient un exposé de deux heures devant un public passif et choqué par la brutalité du récit. Il décide alors d'élaborer une méthode pédagogique qui encourage le visiteurs à découvrir le site avec davantage d'interactivité, à penser sa signification aujourd'hui, et à développer un savoir historique de manière autonome. La tâche du guide-transmetteur est ic limitée à « aider les visiteurs à décrypter ce qu'ils voient » et à stimuler la discussion. Laceès direct a a des source documentaires (des photos et de témoignages ecrits), puis lobservation des lieux s'averent essentiels. Comme Heidemarie Uhl, l'auteur souligne d'ailleurs l'importance de déconstruire certains mythes - le « mythe victimair autrichien", en loccurrence - et d'illustrer notamment à quel degréle camp de Mauthausen faisait partie intégrante de la société autrichienne de l'époque, et de la vie des villageois. rique. Le sujet post-mémoriel n'est $n$ témoin ni archiviste, mais un enquêteu qui explore des images « orphelines (sans témoin), trouvées sur le Net, et qui renvoient donc à un passé qu'il n’a pas vécu. Ainsi, l'oubli contre leque le sujet post-mémoriel lutte ne provient pas d'une raréfaction des documents du passé, mais au contraire, de leur abondance. À travers l'analyse de trois productions (une bande dessinée, un roman-enquête et un film), Sébastien Fevry montre comment les différents auteurs ne « se souviennent » pas au sens litteral, mais reagencent et recontextualisent des images anonymes pour les insérer dans une véritable dynamique mémorielle.

L’avenir de la mémoire, incontestablement, dépendra largement de notre capacité à repenser les principes de base et les stratégies du travail de mémoire tel qu'il a été conçuà la fin de la Seconde Guerre mondiale. Les derniers témoin directs de la Shoah disparaissent, ceux qui pendant des décennies nous on appelé à la vigilance en partageant leurs histoires douloureuses avec un inlassable dévouement.

Les contributions de ce dossie nous indiquent que dans l'avenir, mémoire sera transnationale etcritique, dénuée de mythes, et s'appuiera sur de nouvelles pédagogies de transmission, de nouvelles figures mémorielles, et d nouvelles façons de remémorer.

Anneleen Spiessen le lieu commun de la « disparition des témoins » signale en réalite le désarro de ne pas avoir trouvé de modèle efficace pour la transmission d une experience radicale et collective. Primo Levilui- même, dans Les Naufragés et les res-
Le « sujet post-mémoriel », don Sébastien Fevry dessine ensuite le contours, constitue une autre réponse à la question de la disparition des témoins et offre de nouvelles perspec- tives de transmission dans l'ère numé- demain, nous serons [1998], P. 